\title{
Gestational weight gain and gestational diabetes among Emirati and Arab women in the United Arab Emirates: results from the MISC cohort
}

Mona Hashim ${ }^{1 \dagger}$, Hadia Radwan ${ }^{1+}$, Hayder Hasan ${ }^{1}$, Reyad Shaker Obaid ${ }^{1}$, Hessa Al Ghazal ${ }^{2}$, Marwa Al Hilali Rana Rayess ${ }^{1}$, Noor Chehayber ${ }^{1}$, Hamid Jan Jan Mohamed ${ }^{4+}$ and Farah Naja ${ }^{5^{*+}}$

\begin{abstract}
Background: Nutritional status of women during pregnancy has been considered an important prognostic indicator of pregnancy outcomes.

Objectives: To investigate the pattern of gestational weight gain (GWG) and gestational diabetes mellitus (GDM) and their risk factors among a cohort of Emirati and Arab women residing in the United Arab Emirates (UAE). A secondary objective was to investigate pre-pregnancy body mass index (BMI) and its socio-demographic correlates among study participants.

Methods: Data of 256 pregnant women participating in the cohort study, the Mother-Infant Study Cohort (MISC) were used in this study. Healthy pregnant mothers with no history of chronic diseases were interviewed during their third trimester in different hospitals in UAE. Data were collected using interviewer-administered multicomponent questionnaires addressing maternal sociodemographic and lifestyle characteristics. Maternal weight, weight gain, and GDM were recorded from the hospital medical records.
\end{abstract}

Results: Among the study participants, 71.1\% had inadequate GWG: 31.6\% insufficient and 39.5\% excessive GWG. 19.1\% reported having GDM and more than half of the participants (59.4\%) had a pre-pregnancy BMI $\geq 25 \mathrm{~kg} / \mathrm{m}^{2}$. The findings of the multiple multinomial logistic regression showed that multiparous women had decreased odds of excessive gain as compared to primiparous [odds ratio (OR): $0.17 ; 95 \% \mathrm{Cl}: 0.05-0.54$ ]. Furthermore, women with a pre-pregnancy BMI $\geq 25$ $\mathrm{kg} / \mathrm{m}^{2}$ had increased odds of excessive gain (OR: 2.23; 95\%Cl: 1.00-5.10) as compared to those with pre-pregnancy BMl < $25 \mathrm{~kg} / \mathrm{m}^{2}$. Similarly, women who had a pre-pregnancy BMl $\geq 25 \mathrm{~kg} / \mathrm{m}^{2}$ were at higher risk of having GDM (OR: 2.37; 95\%Cl: 1.10-5.12). As for the associations of women's characteristics with pre-pregnancy BMI, age and regular breakfast consumption level were significant predictors of higher pre-pregnancy BMI.

\footnotetext{
* Correspondence: fn14@aub.edu.lb

${ }^{\dagger}$ Mona Hashim, Hadia Radwan, Hamid Jan Jan Mohamed and Farah Naja contributed equally to this work.

${ }^{5}$ Department of Nutrition and Food Sciences, American University of Beirut, Beirut, Lebanon

Full list of author information is available at the end of the article
}

(c) The Author(s). 2019 Open Access This article is distributed under the terms of the Creative Commons Attribution 4.0 International License (http://creativecommons.org/licenses/by/4.0/), which permits unrestricted use, distribution, and reproduction in any medium, provided you give appropriate credit to the original author(s) and the source, provide a link to the Creative Commons license, and indicate if changes were made. The Creative Commons Public Domain Dedication waiver (http://creativecommons.org/publicdomain/zero/1.0/) applies to the data made available in this article, unless otherwise stated. 


\begin{abstract}
(Continued from previous page)
Conclusions: This study revealed alarming prevalence rates of inadequate, mainly excessive, GWG and GDM among the MISC participants. Pre-pregnancy BMI was found a risk factor for both of these conditions (GWG and GDM). In addition, age and regular breakfast consumption were significant determinants of pre-pregnancy BMI. Healthcare providers are encouraged to counsel pregnant women to maintain normal body weight before and throughout pregnancy by advocating healthy eating and increased physical activity in order to reduce the risk of excessive weight gain and its associated complications.
\end{abstract}

Keywords: Gestational weight gain, Gestational diabetes, Pre-pregnancy BMI, UAE,

\section{Background}

Pregnancy is among the most critical periods of development in the human lifespan, whereby exposures during this period are postulated to have lifelong implications on the health of women as well as their offspring. The Developmental Origins of Health and Disease (DoHaD) hypothesis proposed a link between prenatal, perinatal and early postnatal exposure to certain environmental, dietary, and lifestyle factors and subsequent development of obesity and non-communicable diseases [1]. Among lifestyle factors, gestational weight gain (GWG) has been identified as a major predictor of obstetric, neonatal outcomes as well as health later in life [2].

The influence of GWG on pregnancy complications and outcome depend on pre-pregnancy BMI, hence the Institute of Medicine (IOM) has set the guidelines for GWG according to pre-pregnancy BMI [2]. Previous studies have shown that adequate pregnancy weight gain is crucial for optimal outcomes for both mothers and infants [3, 4]. More specifically, restricted GWG lower than the recommendations were found to be associated with stillbirth, infant death, and child neurocognitive development and behavior [5]. On the other hand, excessive GWG during early pregnancy was associated with an increased risk of gestational hypertension and preeclampsia, caesarean delivery, macrosomia, and postpartum weight retention after delivery [6-8]. Furthermore, excessive GWG was found to exacerbate the generational impact of obesity, whereby women who exceed the recommended weight gain during pregnancy are more likely to retain weight post-partum and to enter the next pregnancy with a higher BMI and deliver heavier babies, who have higher odds to become overweight or obese adults later in life [9].

A common complication associated with excessive weight gain during pregnancy is gestational diabetes mellitus (GDM), characterized by glucose intolerance, of variable degrees, with an onset first recognized during pregnancy [10]. A plethora of literature documented the adverse health implications of GDM for both mother and child. Although glucose homeostasis normalises shortly after delivery, a woman with GDM remains at a higher risk to develop type 2 diabetes mellitus (T2DM) later in life [11], increasing her predisposition to cardiovascular, renal and retinal diseases. In fact, the results of a meta-analysis showed that the relative risk to develop T2DM among women with GDM was 7.7, 95\%CI (4.79-11.51) compared to those who had a normoglycemic pregnancy [12]. With regards to the health of the newborn, GDM was found to increase the risk of fetal macrosomia by $15-45 \%$, if the baby was born to a mother with GDM compared to a mother with normal glucose homeostatis [13]. A baby who is born with a weight greater than the 90th percentile or above $4000 \mathrm{~g}$ is typically considered a microsomic baby [14]. Macrosomia is associated with numerous fetal compalications such as shoulder dystocia, perinatal asphyxia, hyperinsulinemia, neonatal jaundice and neonatal morbidity $[11,13]$.

In light of the significant health implications of GWG and GDM on the health of the mother and child, it is critical to understand their prevalence and determinants among various populations in order to develop evidence-based interventions and inform public health policies. Most of the current evidence on GWG and GDM stems from studies conducted in Western countries making it difficult to compare or generalize the findings to other parts of the world. Few studies have evaluated patterns of weight gain, GDM correlates and pre-pregnancy BMI in countries where malnutrition and poor weight gain, as well as maternal obesity, are known to coexist [15-18].

The UAE is an economically fast-growing country in population size and per-capita income [19]. The rapid modernization and economic growth provoked a shift in diet and lifestyle factors which triggered a marked increase in the prevalence of overweight and obesity and metabolic abnormalities $[19,20]$. A recent report by Yusufali et al., showed that 41.9 and $19.6 \%$ of women in the UAE were overweight and obese respectively [21].

To the best of our knowledge, there is little known about the patterns of GWG and GDM in the UAE. The main objective of this study was to investigate the pattern of GWG and GDM and their risk factors among a cohort of Emirati and Arab women residing in the UAE. A secondary objective was to investigate pre-pregnancy BMI and its sociodemographic correlates among study participants. 


\section{Methodology}

Data for this study were drawn from the Mother-Infant Study Cohort (MISC), a prospective cohort study that included 256 pregnant women from the UAE. Detailed descriptions of the study methods and the recruitment were previously published [22]. Pregnant women in their third trimester were selected using a convenient sampling approach from three main public governmental hospitals, and seven Primary Health Care (PHC) clinics and Mother and Child Centers $(\mathrm{MCH})$ in the Emirates of Sharjah, Dubai, and Ajman. Subjects' recruitment took place during the period from December 2015 to December 2017. In the MISC, data collection is planned at six time points (third trimester, at delivery, 2, 6, 12 and 24 months postpartum). For the purpose of this study, data pertinent to the first time point (third trimester) were used.

Ethical approvals were obtained from all ethics boards overseeing conduct of research in the Emirates of Dubai, Ajman, and Sharjah including Research and Ethics Committee at the University of Sharjah (REC/14/01/1505), Al Qassimi Clinical Research Centre Ethical Research Committee (REC Reference Number: $21512015 \pm 03$ ), Ministry of Health Ethical Research Committee (R02), and Dubai Health Authority (DSREC-0/2016). Before enrollment in the study, participating women provided written informed consent.

The inclusion criteria were Emirati and Arab expatriate pregnant women within their 3rd trimester (27-42 weeks of gestation), aged 19 to 40 years old; with singleton pregnancy, free of chronic diseases such as (diabetes, hypertension, kidney disease, and cancer), and not planning to leave the UAE for the duration of the study. Exclusion criteria were: pregnant women with multiple pregnancies and those who were diagnosed as high-risk pregnancy or had a history of chronic diseases.

In the clinics/hospitals, data was collected using interviewer-administered multi-component questionnaires addressing maternal sociodemographic and lifestyle characteristics such as age (in years), nationality (Emirati or Arab), occupation (employed versus housewife), education (intermediate or less, high school/technical diploma and university), parity (primiparous versus multiparous), income, parity, daily breakfast consumption (daily versus breakfast skippers), and physical activity. The latter was assessed using the Pregnancy Physical Activity Questionnaire (PPAQ) [23], whereby total physical activity was calculated by weighting each type of activity by its energy requirements defined in The metabolic equivalent of task (MET) (multiples of the resting metabolic rate for an activity multiplied by the minutes performed). Based on METS-min per week, three categories of physical activity were assigned, including low, moderate, and high intensity.

\section{Gestational diabetes mellitus (GDM)}

The clinical diagnosis of GDM was obtained from the clinical record. Pregnant women participating in this study were screened for GDM during their 24-28 weeks of gestation using the National Institute for Health and Care Excellence (NICE) Diabetes in Pregnancy criteria [24].

\section{Maternal pre-pregnancy body mass index (BMI)}

Maternal pre-pregnancy BMI referred to BMI before pregnancy. The latter was calculated using height and pre-pregnancy weight. Mother height was obtained during the visit using standard protocol and was measured to the nearest $0.1 \mathrm{~cm}(\mathrm{~cm})$ using Seca 220 Telescopic Measuring Rod for Column Scales. (As for the weight before pregnancy, it was extracted from the medical record. In this record, women were asked to report their last weight before pregnancy which was recorded during the first antenatal visit. BMI was calculated as weight (in $\mathrm{kg}$ ) divided by square height (in meter). Then the prepregnancy BMI was categorized according to the World Health Organization (WHO) classification [25]: BMI less than $18.5 \mathrm{~kg} / \mathrm{m}^{2}$ as underweight, BMI 18.5 to $24.9 \mathrm{~kg} / \mathrm{m}^{2}$ as normal weight, BMI 25.0 to $29.9 \mathrm{~kg} / \mathrm{m}^{2}$ as overweight, and BMI $30.0 \mathrm{~kg} / \mathrm{m}^{2}$ or greater as obese.

\section{Gestational weight Gain}

GWG was calculated as the difference between the recorded pre-pregnancy weight of the mother and the last weight measured before delivery which was derived from the medical records. Further, GWG was categorized as having gained insufficient, adequate, or excessive weight relative to their pre-pregnancy BMI according to the IOM guidelines. Accordingly, adequate GWG was a function of pre pregnancy BMI. Inadequate weight gain was defined as the gestational weight gain either above or below the IOM recommendations These guidelines recommend that women who are underweight should gain 12.5 to $18 \mathrm{~kg}$, women of normal weight should gain 11.5 to $16 \mathrm{~kg}$, women who are overweight should gain 7 to $11.5 \mathrm{~kg}$, and obese women should gain 5 to $9 \mathrm{~kg}$ over the course of their pregnancy [2].

\section{Statistical analysis}

Participants' characteristics were presented as means \pm standard deviation (SD) and proportions for continuous and categorical variables respectively $(n=$ 256). Simple and multiple multinomial logistic regressions were used to examine the effect of participants' characteristics on GWG. In these regression analyses, GWG was the dependent variable (with normal GWG as the reference category) and participants' characteristics as the independent variables. For the association between these characteristics and GDM, simple and multiple logistic regression analyses were used with 
GDM as the dependent variable. Similarly, simple and multiple logistic regressions were applied for the association between participants' characteristics and prepregnancy $B M I \geq 25 \mathrm{~kg} / \mathrm{m}^{2}$. In all analyses, variables with a $p$ value of $<0.25$ in simple regressions were included in the multiple regression models in addition to age. A $p$-value $<0.05$ was used to indicate statistical significance. The Statistical Package for Social Sciences (SPSS) version 22 (IBM Corp. Released 2013. IBM SPSS Statistics for Windows, Version 22.0. IBM Corp: Armonk, NY, USA) was used for data cleaning, management, and analyses.

\section{Results}

Out of a total of 420 women who were approached to participate in the MISC, 256 women completed visit 1 and were included in the analysis for this study (response rate $61 \%$ ).

Descriptive characteristics of the study participants are presented in Table 1 . The average age of study participants was $30.5 \pm 6.0$ years, and the majority $(53.5 \%)$ were $\geq$ 30 years. The study sample included more Arab women then Emirati (59\% versus $41 \%$ ). Only $13.7 \%$ of the participants have an intermediate or lower education level, while $54.7 \%$ had a high school or diploma and $31.6 \%$ were holders of a university degree. The majority of women were housewives $(82.4 \%)$ and the monthly family income exceeded 10,000 AED (UAE currency; 1 US \$ = 3.67 AED) for $53.4 \%$ of women and only $9.3 \%$ had an income of > 5000 AED for $53.4 \%$ of women and only $9.3 \%$ fell below 5000 AED. Almost three in four women participated in this study were multiparous (76.6\%). A considerable proportion of women had low-intensity physical activity $(64.8 \%)$ and consumed breakfast daily (69.9\%). With regards to pre-pregnancy BMI, more than half of the participants $(59.4 \%)$ had a BMI $\geq 25 \mathrm{~kg} / \mathrm{m}^{2}$. Among study participants, $71.1 \%$ had inadequate GWG: 31.6\% insufficient and 39.5\% excessive GWG. Furthermore, 19.1\% reported having GDM (Table 1).

The associations of various characteristics of study participants with GWG were examined using simple and multiple multinomial logistic regression (Table 2). For these associations, the adequate GWG was used as reference category. The results of the simple regression showed that both parity and pre-pregnancy BMI were significantly associated with GWG. Multiparous women were less likely to have excessive GWG $(p<0.05)$. Women with a pre-pregnancy BMI $\geq 25 \mathrm{~kg} / \mathrm{m}^{2}$ had higher odds of gaining excessive weight during their pregnancy $(p<0.05)$ (Table 2). These findings were confirmed by those of the multiple multinomial logistic regression, after adjustment for age. More specifically multiparous women were $83 \%$ less likely to have excessive weight gain as compared to primiparous (OR: 0.17 ; $95 \% \mathrm{CI}$ : $0.05-0.54)$. Furthermore, women with a pre-pregnancy
Table 1 Descriptive characteristics of pregnant women participating in this study $(n=256)$

\begin{tabular}{ll}
\hline & Total $^{\mathrm{a}}$ \\
\hline Mother's Age (years) & $30.5 \pm 6.0$ \\
$18-24.9$ & $57(22.3)$ \\
$25-29.9$ & $62(24.2)$ \\
$\geq 30$ & $137(53.5)$ \\
Nationality & \\
Emirati $_{\text {Arab }}^{\text {b }}$ & $105(41.0)$ \\
\end{tabular}

Education

Intermediate or less

35 (13.7)

High School/ Technical Diploma

$140(54.7)$

University

$81(31.6)$

Employment Status

Employee

45 (17.6)

Housewife

$211(82.4)$

Family Monthly Income (AED)

$<5000$

$18(9.3)$

5000-10,000

$72(37.3)$

$>10,000$

$103(53.4)$

Parity

Primiparous

$60(23.4)$

Multiparous

$196(76.6)$

Physical Activity (METs)

Low-Intensity Activity

$166(64.8)$

Moderate / High-Intensity Activity

$90(35.2)$

Daily Breakfast Consumption

No

77 (30.1)

Yes

$179(69.9)$

Pre-pregnancy BMI $\left(\mathrm{kg} / \mathrm{m}^{2}\right)^{\mathrm{c}}$

$27.3 \pm 6.2$

$\mathrm{BMl}<25$

$104(40.6)$

$\mathrm{BMI} \geq 25$

$152(59.4)$

Gestational weight gain (GWG) ${ }^{\text {d }}$

Insufficient 81 (31.6)

Adequate $74(28.9)$

Excessive 101 (39.5)

Gestational diabetes mellitus (GDM)

No

$207(80.9)$

Yes

49 (19.1)

${ }^{a}$ Values in the table are presented as $\mathrm{n}(\%)$ for categorical variables and mean (SD) for continuous variables

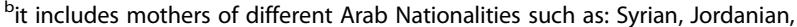
Sudanese, Palestinian, Lebanese, and Algerians etc. ...

${ }^{c}$ According to WHO classification 2007; ${ }^{d}$ According to IOM classification 2009

$\mathrm{BMI} \geq 25 \mathrm{~kg} / \mathrm{m}^{2}$ had 2.23 times the odds of excessive GWG as compared to those $<25 \mathrm{~kg} / \mathrm{m}^{2}$ (OR: 2.23 ; $95 \%$ CI: 1.00 5.10) (Data not shown). 
Table 2 Simple multinomial regression analysis for the association of subjects' characteristics with gestational weight gain (GWG)

\begin{tabular}{|c|c|c|}
\hline & Insufficient & Excessive \\
\hline & OR $(95 \% \mathrm{Cl})$ & OR $(95 \% \mathrm{Cl})$ \\
\hline \multicolumn{3}{|l|}{ Mother's Age (years) } \\
\hline $18-24.9$ & Ref & Ref \\
\hline $25-29.9$ & $0.64(0.26,1.58)$ & $0.64(0.26,1.57)$ \\
\hline$\geq 30$ & $0.68(0.31,1.53)$ & $0.95(0.43,2.07)$ \\
\hline \multicolumn{3}{|l|}{ Nationality } \\
\hline Emirati & Ref & Ref \\
\hline Arab & $0.95(0.501,1.79)$ & $1.15(0.62,2.11)$ \\
\hline \multicolumn{3}{|l|}{ Education } \\
\hline Intermediate or less & Ref & Ref \\
\hline High School/ Technical Diploma & $1.80(0.65,5.00)$ & $0.96(0.41,2.26)$ \\
\hline University & $2.57(0.86,7.65)$ & $1.16(0.46,3.00)$ \\
\hline \multicolumn{3}{|l|}{ Employment Status } \\
\hline Employee & Ref & Ref \\
\hline Housewife & $0.80(0.36,1.79)$ & $1.22(0.54,2.75)$ \\
\hline \multicolumn{3}{|l|}{ Family Monthly Income (AED) } \\
\hline$<5000$ & Ref & Ref \\
\hline $5000-10,000$ & $0.40(0.10,1.71)$ & $0.79(0.18,3.54)$ \\
\hline$>10,000$ & $0.28(0.07,1.15)$ & $0.64(0.15,2.74)$ \\
\hline \multicolumn{3}{|l|}{ Parity } \\
\hline Primiparous & Ref & Ref \\
\hline Multiparous & $0.53(0.24,1.20)$ & $0.43(0.20,0.94)$ \\
\hline \multicolumn{3}{|l|}{ Physical Activity (METs) } \\
\hline Low-Intensity Activity & Ref & Ref \\
\hline Moderate /High Intensity Activity & $0.69(0.36,1.34)$ & $0.74(0.40,1.39)$ \\
\hline \multicolumn{3}{|l|}{ Daily Breakfast Consumption } \\
\hline No & Ref & Ref \\
\hline Yes & $0.905(0.45,1.77)$ & $1.05(0.54,2.03)$ \\
\hline \multicolumn{3}{|l|}{ Pre-pregnancy BMI ${ }^{\mathrm{a}}$} \\
\hline $\mathrm{BMI}<25$ & Ref & Ref \\
\hline $\mathrm{BMI} \geq 25$ & $0.615(0.33,1.16)$ & $2.88(1.502,5.534)$ \\
\hline \multicolumn{3}{|l|}{ Gestational Diabetes } \\
\hline No & Ref & Ref \\
\hline Yes & $1.30(0.56,3.05)$ & $1.69(0.77,3.73)$ \\
\hline
\end{tabular}

The reference category is 'Adequate'

Analysis Of Variance (ANOVA) showed no significant difference among the absolute GWG (expressed in $\mathrm{Kg})$ for underweight $\left(<18.5 \mathrm{~kg} / \mathrm{m}^{2}\right)$, normal $(18.5-24.9$ $\left.\mathrm{kg} / \mathrm{m}^{2}\right)$, overweight $\left(25-29.9 \mathrm{~kg} / \mathrm{m}^{2}\right)$ and obese $(\geq 30$ $\left.\mathrm{kg} / \mathrm{m}^{2}\right)(13.45 \pm 6.43,12.53 \pm 6.09,12.50 \pm 7.78,10.34 \pm$ 8.28 respectively, $p>0.05)$. The distribution of the various categories of GWG (insufficient, adequate and excessive), according to pre-pregnancy BMI were depicted in Fig. 1. While the proportions of insufficient GWG decreased across increasing pre-pregnancy BMI, those of excessive GWG increased. Proportions of women with insufficient GWG were 63.6, 43, 22.4, and $22.4 \%$, while the proportions of women with excessive GWG were 18.2, 22.6, 53.9 and $48.7 \%$ among women who were underweight, normal, overweight and obese before pregnancy respectively (Fig. 1). It is important to note that the highest proportion of women with adequate GWG was among those with a normal prepregnancy BMI (34.4\% among women with a normal pre-pregnancy BMI versus 18.2, 23.7 and $28.9 \%$ who 


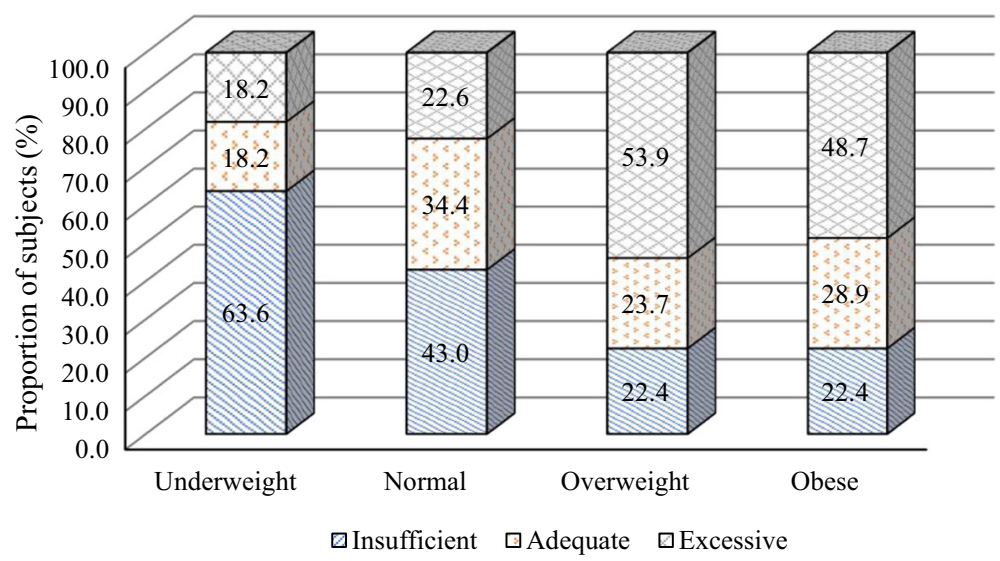

Fig. 1 Comparison of weight gain across different body mass index categories

were underweight, overweight and obese before pregnancy, respectively).

As for the associations of subjects' characteristics with GDM, the results of the simple logistic regression indicated that older age ( $\geq 30$ years), daily breakfast intake, and prepregnancy BMI $\left(\geq 25 \mathrm{~kg} / \mathrm{m}^{2}\right)$ were associated with higher odds of GDM $(p<0.05)$ (Table 3). After age-adjustment, only pre-pregnancy BMI was found to be associated with GDM, whereby, women with a pre-pregnancy BMI $\geq 25 \mathrm{~kg} /$ $\mathrm{m}^{2}$ had 2.37 times the odds of having GDM, as compared to those with pre-pregnancy BMI $<25 \mathrm{~kg} / \mathrm{m}^{2}$ (OR: 2.37 ; 95\%CI: 1.10-5.12) (Data not shown).

Simple and multiple logistic regression analyses were used to investigate the associations of subjects' characteristics with pre-pregnancy BMI. The results of the simple regression showed that age, education level, parity as well as daily breakfast intake were significantly associated with pre-pregnancy BMI (Table 4). After adjustment, only age and breakfast consumption maintained significant associations. Compared to women aged 1824.9 years, those 30 years of age or older had significantly higher odds of having a pre-pregnancy BMI $\geq 25 \mathrm{~kg} / \mathrm{m}^{2}$ (OR: 4.75; 95\%CI: 1.85-12.20). Furthermore, subjects who consumed breakfast regularly were 2.21 more likely to have a pre-pregnancy BMI $\geq 25 \mathrm{~kg} / \mathrm{m}^{2}$, as compared to those who did not consume breakfast regularly (OR: 2.21, 95\%CI: 1.10-4.44) (Data not shown).

\section{Discussion}

This is the first study to report results from the MISC cohort, one of a few mother and child cohorts in the Middle East and North Africa (MENA). The study investigated GWG, GDM and their correlates among the MISC participants, a cohort of Emirati and Arab women residing in the UAE, and identified the sociodemographic correlates of pre-pregnancy BMI. The main findings of this study included alarming prevalence of inadequate GWG (insufficient and excessive) and of GDM. Furthermore, while parity and pre-pregnancy BMI were found to be predictors of excessive GWG, only pre-pregnancy BMI was associated with higher odds of GDM. Pre-pregnancy overweight and obesity were correlated with a higher mother's age and regular breakfast consumption.

A main finding of this study was the significant prevalence of insufficient and excessive GWG that was observed among the participants, with only $30 \%$ of participants falling within the adequate GWG as per the IOM guidelines. More specifically, 32and 39\% had insufficient and excessive GWG, respectively. These rates are within the range of those reported by a recent systematic review and meta-analysis aimed to examine GWG and which included 23 studies, with a total sample size of 1309 pregnant women. The results of this review indicated that 23 and $47 \%$ had insufficient and excessive GWG, respectively [8] . The high prevalence of inadequate GWG and specifically the excessive GWG found in this study is of public health concern, especially in the light of mounting evidence for its impact not only on the birth outcome but also on disease risk later in life $[26,27]$. A more direct implication of the excessive GWG among pregnant women is the higher risk of these women to retain weight and become overweight and obese, especially following more than one pregnancy. In fact, women who gained excessive weight during pregnancy were 3.2 times more likely to retain their weight postpartum, and twice as likely to retain at least $5 \mathrm{~kg}$ of weight post-pregnancy [28]. The direct effect of excessive GWG on obesity is critical, especially in the context of the UAE, where obesity prevalence rates are soaring [29]. These findings call for a concerted action among various concerned health authorities for proper prenatal nutritional counseling and early interventions to target pregnant who are at risk of inadequate GWG. Therefore, 
Table 3 Simple logistic regression analysis for the associations of subjects' characteristics with Gestational Diabetes

\begin{tabular}{|c|c|}
\hline & OR $(95 \% \mathrm{Cl})$ \\
\hline \multicolumn{2}{|l|}{ Mother's Age (years) } \\
\hline $18-24.9$ & Ref \\
\hline $25-29.9$ & $1.44(0.48,4.35)$ \\
\hline$\geq 30$ & $2.81(1.11,7.11)$ \\
\hline \multicolumn{2}{|l|}{ Nationality } \\
\hline Emirati & Ref \\
\hline Arab & $0.91(0.49,1.71)$ \\
\hline \multicolumn{2}{|l|}{ Education } \\
\hline Intermediate or less & Ref \\
\hline High School/ Technical Diploma & $1.37(0.49,3.86)$ \\
\hline University & $1.71(0.58,5.06)$ \\
\hline \multicolumn{2}{|l|}{ Employment Status } \\
\hline Employee & Ref \\
\hline Housewife & $0.59(0.28,1.24)$ \\
\hline \multicolumn{2}{|l|}{ Family Monthly Income (AED) } \\
\hline$<5000$ & Ref \\
\hline $5000-10,000$ & $1.55(0.40,5.98)$ \\
\hline$>10,000$ & $1.28(0.34,4.84)$ \\
\hline \multicolumn{2}{|l|}{ Parity } \\
\hline Primiparous & Ref \\
\hline Multiparous & $1.07(0.51,2.25)$ \\
\hline \multicolumn{2}{|l|}{ Physical Activity (METs) } \\
\hline Low-Intensity Activity & Ref \\
\hline Moderate /High Intensity Activity & $0.98(0.51,1.88)$ \\
\hline \multicolumn{2}{|l|}{ Daily Breakfast Consumption } \\
\hline No & Ref \\
\hline Yes & $2.56(1.14,5.77)$ \\
\hline \multicolumn{2}{|l|}{ Pre-pregnancy BMI } \\
\hline $\mathrm{BMI}<25$ & Ref \\
\hline $\mathrm{BMI} \geq 25$ & $2.82(1.37,5.82)$ \\
\hline
\end{tabular}

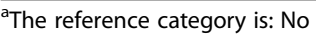

identifying the context specific correlates of inadequate GWG is important to control GWG.

In this study, excessive GWG was found to be associated with parity and pre-pregnancy BMI. With regards to parity, primiparous women were more likely to gain excessive weight compared to multiparous participants. Findings of this study were in accordance with other investigations that reported primiparous women gaining more pregnancy weight or were more likely to exceed GWG recommendations than multiparous women [30-32]. It is arguable that women with more children spend less time at rest and are more likely to be active looking after existing children as compared to women with no
Table 4 Simple logistic regression analysis for the association of study characteristics with pre-pregnancy BMI (BMI $\geq 25)$

\begin{tabular}{ll}
\hline & OR $(95 \% \mathrm{Cl})$ \\
\hline Mother's Age (years) & \\
$18-24.9$ & Ref \\
$25-29.9$ & $1.85(0.89,3.87)$ \\
$\geq 30$ & $5.19(2.67,10.08)$ \\
Nationality & \\
Emirati & Ref \\
Arab & $1.02(0.62,1.70)$ \\
Education & \\
Intermediate or less & Ref \\
High School/ Technical Diploma & $38(.16,0.90)$ \\
University & $0.90(0.16,0.96)$ \\
Employment Status & \\
Employee & Ref \\
Housewife & $1.50(0.79,2.87)$ \\
Family Monthly Income (AED) & \\
$<5000$ & Ref \\
5000-10,000 & $2.08(0.72,6.00)$ \\
$>10,000$ & $2.48(0.89,6.91)$ \\
Parity & Ref \\
Primiparous & $1.94(1.13,3.34)$ \\
Multiparous & \\
Physical Activity (METs) & \\
Low-Intensity Activity & $(1.20,3.87)$ \\
Moderate /High Intensity Activity & \\
Daily Breakfast Consumption & \\
No & \\
Yes & \\
\hline
\end{tabular}

children [33]. As for the association between prepregnancy BMI and GWG, the results of this study indicated that being overweight or obese prior to pregnancy significantly increased the odds of excessive GWG. Consistent with our findings, Weisman et al., in a study among 103 pregnant women in the USA, reported that being overweight or obese substantially increases the odds of gaining excessive weight [34]. Furthermore, Deputy et al. showed that pre-pregnant overweight and obese women were about 2 and 3 times respectively more likely to have excessive weight gains above the IOM recommendations [35]. Begum et al., indicated that higher prepregnancy BMI is a significant predictor of excessive weight gain during pregnancy; $80 \%$ of the overweight or obese pregnant women gained weight more than the recommended value [36]. A possible explanation for this phenomena is that overweight or obese 
mothers may have a high energy diet and low levels of physical activity during their pregnancy which may lead them to gain excessive weight $[37,38]$. In light of the study findings regarding the correlates of inadequate GWG, specific nutrition and lifestyle counselling interventions are encouraged to target primiparous as well as overweight and obese women during antenatal care visits to prevent further increase in weight during pregnancy. It is important to note, however, that the absolute GWG values did not vary significantly among the various categories of pre pregnancy BMI. The association between GWG classification and pre pregnancy BMI could have been confounded by the different targets/recommendations of weight gain for each BMI category.

In addition to GWG, this study aimed to examine GDM and its correlates among the MISC participants. The findings of this study showed an alarmingly high prevalence of GDM (19\%). Previous studies in the UAE reported that GDM prevalence varied between 7.9 and 24.9\% [39]. A few studies reported similar and even higher prevalence rates. For instance, in Vietnam and in Singapore, the prevalence of GDM was 20.06 and $18.93 \%$, respectively [40]. Furthermore, in a cohort study in Saudi Arabia, a higher GDM percentage was reported among 2354 participants (24.2\%) [41]. However these prevalence rates are higher than those reported by other Gulf countries, $(4.2 \%$ in Oman, $16.3 \%$ in Qatar, and $10.1 \%$ in Bahrain) [42], and also higher than the median prevalence of GDM estimate obtained by a recent review in the MENA (12.9\%) [43]. Moreover, lower GDM rates were reported in other parts of the world; by some Asian countries where the prevalence of GDM among Korean mothers was 4.5, and 6.2\% among Chinese [44]. Similarly, in Europe, lower GDM occurrence was reported from Epifane, a French birth cohort, where $7.7 \%$ of the women had GDM [45]. In Italy, a prospective study that included 14,109 women, GDM was diagnosed in 360 women (2.6\%) [46]. As such, the high prevalence of GDM in the UAE raises major public health concern, especially given the mounting evidence for its association with maternal and neonatal complications during pregnancy as well as adverse health outcomes for both mothers and their newborns [47]. GDM is considered to reflect the underlying T2DM epidemic since many of the women with a history of GDM may be imposed to a sevenfold increased risk of T2DM in later life [12]. This adds to the growing burden of diabetes risk among the population in the UAE. The latter has one of the world's highest prevalence rates of T2DM of $18.7 \%$ and is expected to reach $21.4 \%$ by 2030 [47].

The high prevalence of GDM reported in this study as well as other reports from the UAE, and its significant health sequalae underscores the need to investigate the determinant of GDM. The results of the multinomial logistic regression in this study showed a significant association between pre-pregnancy BMI and the incidence risk of GDM. The participants with a pre-pregnancy BMI $\geq 25 \mathrm{~kg} / \mathrm{m}^{2}$ were over two times more likely to have GDM, as compared to those with pre-pregnancy BMI < $25 \mathrm{~kg} / \mathrm{m}^{2}$. A meta-analysis based on 31 cohort studies with 364,668 subjects found that women with obesity had higher odds of developing GDM compared to women of normal weight [OR of 3.76(3.31-4.28)]. Other studies also reported similar findings [48].

Another study reported similar findings, whereby mothers with pre-pregnancy overweight or obesity had a 2.19 fold the risk of developing GDM [44]. Together these aforementioned studies confirmed that pregestational obesity is an independent risk factor for GDM [49-52]. In a previous study in the UAE, a higher risk of GDM increased almost 4 times (OR 3.75, 95\% CI, 1.83$7.69, p=0.001$ ) in the morbidly obese group in comparison to controls [53]. Maternal obesity is consistently pointed out as a major and modifiable risk factor for GDM [54]. Since GDM and obesity are frequently comorbid conditions, it is well established that women who were diagnosed with GDM during pregnancy, their pre-pregnancy BMI was shown to increase the risk of prediabetes and diabetes later in life $[7,55]$. It has been estimated that compared to women with a normal BMI, the risk of pregnancy diabetes is 2 and 4 times higher in overweight and obese women, respectively [50].

The association between a higher pre-pregnancy BMI and GDM might be explained by the fact that obese women, due to greater fat deposition, have lower insulin sensitivity as compared normal weight women [56]. Prevention of GDM is considered a key strategy for breaking the intergenerational cycle of obesity and diabetes [57]. Thus, strategies aiming at preventing obesity in young women and keeping appropriate preconception weight in pre-pregnant women are essential for the prevention of GDM.

Given that Pre-pregnancy BMI has emerged as a strong predictor of GWG and GDM, it was addressed as a secondary objective for this study inorder to identify the sociodemographic correlates of pre-pregnancy BMI among the participants. More than half of the participants in our study were either overweight or obese before pregnancy. This is consistent with other investigators $[15,17]$. While other studies reported lower prevalence rates of maternal pre-pregnancy BMI overweight and obesity among their pregnant participants, where about one-third of women were either overweight or obese [58-60].

In this study, maternal age and breakfast consumption were found to be significantly associated with prepregnancy BMI. Pre-pregnancy BMI was significantly higher among women who were older and who consumed 
breakfast regularly. With regard to age, many studies had reported that mothers who were older in age were more likely to be obese before pregnancy $[6,61]$. For instance, Boudet-Berquier et al. found that women aged 30-34 years, were more likely to have obesity before pregnancy than to be of normal weight [45]. A possible explanation could lay in the fact that as the mother increases in age, their physical activity and energy expenditure levels decrease [62, 63]. Furthermore, an older age is most likely to be accompanied by multiple pregnancies and child birth which could result in weight retention and development of overweight and obesity. Very few studies examined the association between breakfast consumption and pre pregnancy BMI. Contrary to our findings, a study among Korean pregnant women showed that regular breakfast consumption was more prevalent among women with a normal or underweight pre pregnancy BMI as compared to those with overweight or obese pre pregnancy BMI [64]. In this context, it is suggested that energy density and nutrient composition of the breakfast could be an important confounder for the association between the frequency of consumption and pre pregnancy BMI. More specifically, a breakfast high in simple as opposed to complex carbohydrates tend to be associated with a higher BMI, whereas a breakfast richer in protein, fruits and vegetables could be associated with lower BMI [65]. It is therefore recommended that future studies examining the association between breakfast consumption and pre pregnancy BMI take into consideration energy and nutrient composition of the breakfast in addition to its frequency.

\section{Strengths and limitations}

To our knowledge, this is the first study which investigated GWG, GDM and its correlates and the sociodemographic factors related to pre-pregnancy BMI in the UAE. Moreover, it was the first to use the international IOM recommendations to examine GWG in the UAE. Besides the publication that described the protocol of the MISC [22], this manuscript is the first to report on the results of this cohort. The latter being one of few cohorts in the region investigating early metabolic programming of lifelong health to facilitate identifying atrisk women and developing tailored interventions.

We are aware of important limitations of this study. First the small sample size could have led to underpowered analyses and a higher risk of type II error, especially in relation to GDM and its determinants. Such a sample size also restricted a more detailed classification of pre pregnancy BMI. Second, the pre-pregnancy BMI, GWG, as well as GDM, were extracted from the participants' records. Although in the clinics and health centers where recruitment took place, standards techniques and guidelines were implemented in obtaining weight and diagnosing GD, it is inevitable that random errors could have occurred. Third, the information collected regarding socio-demographic and lifestyle characteristics was based on subjects' reporting and hence may have been subject to recall error. That said, the field workers were trained to implement standard interviewing techniques with minimal leading questions in order to minimize any interviewer bias or social desirability bias. Fourth, the low response rate observed in this study could potentially lead to selection bias. Lastly, it is recommended that future longitudinal studies investigating the determinants of GDM take into consideration important variables such as family history of diabetes and a history of gestational diabetes in previous pregnancies.

\section{Conclusions}

This study revealed alarming prevalence rates of inadequate, mainly excessive, GWG and GDM among the MISC participants. Pre-pregnancy BMI was found to be a common denominator in the etiology of both excessive GWG and GDM. In addition, age and education were significant determinants of pre-pregnancy BMI.

As such, women of reproductive age with a high BMI should be given additional attention in targeted preconceptional and inter pregnancy interventions in order to prevent GWG and GDM which will prepare them to start the next pregnancy with a healthier BMI. Hence, healthcare specialists are encouraged not only to focus on fetus health during the clinic visit of pregnant mothers but also should pay more attention to maternal health. They should advise them on appropriate weight gain during pregnancy by promoting healthy diet and physical activity in order to prevent GDM and postpartum weight retention and decrease obesity related risks in subsequent pregnancies.

\section{Abbreviations}

ANOVA: Analysis Of Variance; BMI: Body Mass Index; Cl: Confidence Interval; Cm: Centimeters; DoHaD: Developmental Origins of Health and Disease; GDM: Gestational Diabetes Mellitus; GWG: Gestational Weight gain; IOM: Institute of Medicine; MCH: Mother and Child Centers; MENA: Middle East and North Africa; MET: The Metabolic Equivalent of Task; MISC: MotherInfant Study Cohort; NICE: National Institute for Health and Care Excellence; OR: Odds Ratio; PHC: Primary Health Care; PPAQ: Pregnancy Physical Activity Questionnaire; REC: Research and Ethics Committee; SD: Standard Deviation; SPSS: Statistical Package for Social Sciences; T2DM: Type 2 Diabetes Mellitus; UAE: United Arab Emirates; WHO: World Health Organization

\section{Acknowledgments}

We would like to acknowledge all the mothers, nurses, and hospital and health clinic staff as well as the research assistants who participated in this study. Moreover, we would like also to thank Ms. Nada Adra for her technical and statistical assistance.

\section{Authors' contributions}

$\mathrm{MH}, \mathrm{HR}, \mathrm{FN}, \mathrm{HH}, \mathrm{HJJM}, \mathrm{RSO}, \mathrm{HAG}, \mathrm{MH}, \mathrm{RR}, \mathrm{NC}$, contributed to conception and design, acquisition of data. MH,FN,HR were involved in analyzing and interpreting the data. $\mathrm{MH}$ and $\mathrm{HR}$ drafted the manuscript.

$\mathrm{RSO}, \mathrm{HH}, \mathrm{HAG}, \mathrm{MH}, \mathrm{RR}, \mathrm{NC}$ have been involved in reviewing the manuscript. FN and HJJM critically revised the final version of the manuscript and ensured its scientific rigor. All authors have read and approved the final version of 
the manuscript. Each author has participated sufficiently in the work to take public responsibility for appropriate portions of the content; and agree to be accountable for all aspects of the work in ensuring that questions related to the accuracy or integrity of any part of the work are appropriately investigated and resolved.

\section{Funding}

We would like to note that The MISC research study has received funding from Al Jalila Foundation (AJF 201510) and University of Sharjah Collaborative Grant (1501057003-P). The study sponsors had no involvement in the research design, data collection, interpretation of the data, or publication.

\section{Availability of data and materials}

The datasets used and/or analysed during the current study are available from the corresponding author on reasonable request.

\section{Ethics approval and consent to participate}

Ethical approvals were obtained from all ethics boards overseeing conduct of research in the Emirates of Dubai, Ajman, and Sharjah including Research and Ethics Committee at the University of Sharjah (REC/14/01/1505), Al Qassimi Clinical Research Centre Ethical Research Committee (REC Reference Number: 215 12015 \pm 03), Ministry of Health Ethical Research Committee (R02), and Dubai Health Authority (DSREC-0/2016). Before enrollment in the study, participating women provided written informed consent.

\section{Consent for publication}

Not Applicable.

\section{Competing interests}

The authors declare that they have no competing interests.

\section{Author details}

${ }^{1}$ Department of Clinical Nutrition and Dietetics, College of Health Sciences, Research Institute of Medical and Health Sciences (RIMHS), University of Sharjah, Sharjah, United Arab Emirates. 'Family Health Promotion Center, Sharjah, United Arab Emirates. ${ }^{3} \mathrm{Al}$ Qassimi Hospital, Ministry of Health and Prevention, Sharjah, United Arab Emirates. ${ }^{4}$ Nutrition and Dietetics Program, Universiti Sains Malaysia, Kelantan, Malaysia. ${ }^{5}$ Department of Nutrition and Food Sciences, American University of Beirut, Beirut, Lebanon.

Received: 15 October 2018 Accepted: 22 November 2019

Published online: 03 December 2019

\section{References}

1. Kappil M, Wright RO, Sanders AP. Developmental origins of common disease: epigenetic contributions to obesity. Annu Rev Genomics Hum Genet. 2016;17(1):177-92 Available from: http://www.annualreviews.org/ doi/10.1146/annurev-genom-090314-050057.

2. IOM. Nutrition During Pregnancy: Weight Gain, Nutrient Supplements. National Academy Press. Washington: National Academy Press; 1990.

3. Ogunyemi D, Hullett $\mathrm{S}$, Leeper J. Risk A. Prepregnancy body mass index, weight gain during pregnancy, and perinatal outcome in a rural black population. J Matern Fetal Med. 1998;7(4):190-3 Available from: http://www. ncbi.nlm.nih.gov/pubmed/9730485.

4. Merchant SS, Momin IA, Sewani AA, Zuberi NF. Effect of prepregnancy body mass index and gestational weight gain on birth weight. J Pak Med Assoc. 1999;49(1):23-5.

5. Bodnar LM, Siminerio LL, Himes KP, Hutcheon JA, Lash TL, Parisi SM, et al. Maternal obesity and gestational weight gain are risk factors for infant death. Obesity. 2016;24(2):490-8.

6. Yeşilçiçek Çalik K, Korkmaz Yildiz N, Erkaya R. Effects of gestational weight gain and body mass index on obstetric outcome. Saudi J Biol Sci. 2018; 25(6):1085-108. https://doi.org/10.1016/j.sjbs.2018.02.014.

7. Ferraro ZM, Contador F, Tawfiq A, Adamo KB, Gaudet L. Gestational weight gain and medical outcomes of pregnancy. Obstet Med. 2015;8(3):133-7. https://doi.org/10.1177/1753495X15591320.

8. Goldstein RF, Abell SK, Ranasinha S, Misso M, Boyle JA, Black MH, et al. Association of gestational weight gain with maternal and infant outcomes: a systematic review and meta-analysis. JAMA. 2017;317(21):2207-25.
9. Walker R, Bennett C, Blumfield M, Gwini S, Ma J, Wang F, et al. Attenuating pregnancy weight gain - what works and why: a systematic review and meta-analysis. Nutrients. 2018;10(7):4-6.

10. National Diabetes Data Group. Classification and diagnosis of diabetes mellitus and other categories of glucose intolerance. Diabetes. 1995;28(12): 1039-57 Available from: http://diabetes.diabetesjournals.org/content/28/12/ 1039\%5Cnhttp://diabetes.diabetesjournals.org/content/28/12/1039. short\%5Cnhttp://www.ncbi.nlm.nih.gov/pubmed/510803.

11. Kim C, Newton KM, Knopp RH. Gestational diabetes and the incidence of type 2 diabetes: a systematic review. Diabetes Care. 25(10):1862-8 Available from: http://www.ncbi.nlm.nih.gov/pubmed/12351492.

12. Bellamy L, Casas J-P, Hingorani AD, Williams D. Type 2 diabetes mellitus after gestational diabetes: a systematic review and meta-analysis. Lancet (London, England). 2009;373(9677):1773-9 Available from: http://www.ncbi. nlm.nih.gov/pubmed/19465232.

13. Kc K, Shakya S, Zhang H. Gestational diabetes mellitus and macrosomia: a literature review. Ann Nutr Metab. 2015;66:14-20.

14. Surkan PJ, Hsieh CC, Johansson ALV, Dickman PW, Cnattingius S. Reasons for increasing trends in large for gestational age births. Obstet Gynecol. 2004;104(4):720-6.

15. El Rafei R, Abbas HA, Charafeddine L, Nakad P, Al Bizri A, Hamod D, et al. Association of pre-Pregnancy Body Mass Index and Gestational Weight Gain with preterm births and fetal size: an observational study from Lebanon. Paediatr Perinat Epidemiol. 2016;30(1):38-45.

16. Almurshed KS, Bani I A, Al-Kanhal M A, Al-Amri M A. A study of maternal dietary intake during pregnancy in riyadh, saudi arabia. Vol. 14, Journal of family \& community medicine. 2007. p. 9-13. Available from: http://www.pubmedcentral. nih.gov/articlerender.fcgi?artid=3410119\&tool=pmcentrez\&rendertype=abstract

17. Munim S, Maheen H. Association of gestational weight gain and prepregnancy body mass index with adverse pregnancy outcome. J Coll Physicians Surg Pak. 2012;22(11):694-8 Available from: http://www.ncbi.nlm. nih.gov/pubmed/23146848.

18. Yekta Z, Ayatollahi H, Porali R, Farzin A. The effect of pre-pregnancy body mass index and gestational weight gain on pregnancy outcomes in urban care settings in Urmia-Iran. BMC Pregnancy Childbirth. 2006;6:1-8.

19. Ng SW, Zaghloul S, Ali H, Harrison G, Yeatts K, El Sadig M, et al. Nutrition transition in the United Arab Emirates. Eur J Clin Nutr . 2011 Dec [cited 2015 Dec 1];65(12):1328-37. Available from: http://www.pubmedcentral.nih.gov/ articlerender.fcgi?artid=3304306\&tool=pmcentrez\&rendertype=abstract

20. Al Dhaheri AS, Mohamad MN, Jarrar AH, Ohuma EO, Ismail LC, Al Meqbaali FT, et al. A cross-sectional study of the prevalence of metabolic syndrome among young female Emirati adults. PLoS One. 2016;11(7):e0159378. Available from:. https://doi.org/10.1371/journal.pone.0159378.

21. Yusufali A, Bazargani N, Muhammed K, Gabroun A, AlMazrooei A, Agrawal A, et al. NIH Public Access. PLoS One. 2015[cited 2018 Jul 13];10(1):1-8. https:// doi.org/10.1002/ajhb.21014/abstract.

22. Radwan H, Hashim M, Shaker Obaid R, Hasan H, Naja F, Al Ghazal H, et al. The Mother-Infant Study Cohort (MISC): Methodology, challenges, and baseline characteristics. de Souza RJ. PLoS One. 2018;13(5):e0198278. Available from: https://doi.org/10.1371/journal.pone.0198278

23. Chasan-Taber L, Schmidt MD, Roberts DE, Hosmer D, Markenson G, Freedson PS. Development and validation of a pregnancy physical activity questionnaire. Med Sci Sports Exerc. 2004:36(10):1750-60.

24. National Institute for Health and Care Excellence [NICE]. Diabetes in pregnancy: management from preconception to the postnatal period | Guidance and guidelines | NICE. 2015; Available from: https://www.nice.org.uk/guidance/ng3

25. World Health Organization (WHO). Obesity : Preventing and Managing the Global Epidemic. Geneva: WHO Tech Rep Ser; 2000.

26. Jiang H, Qian X, Li M, Lynn H, Fan Y, Jiang H, et al. Can physical activity reduce excessive gestational weight gain? Findings from a Chinese urban pregnant women cohort study. Int J Behav Nutr Phys Act. 2012;9:1-7.

27. Miao M, Dai M, Zhang Y, Sun F, Guo X, Sun G. Influence of maternal overweight, obesity and gestational weight gain on the perinatal outcomes in women with gestational diabetes mellitus. Sci Rep. 2017:7(1):1-8. Available from:. https://doi.org/10.1038/s41598-017-00441-z.

28. Hill B, McPhie $\mathrm{S}$, Skouteris $\mathrm{H}$. The role of parity in gestational weight Gain and postpartum weight retention. Women's Heal Issues. 2016;26(1):123-9. Available from:. https://doi.org/10.1016/j.whi.2015.09.012.

29. Razzak H, El-Metwally A, Harbi A, Al-Shujairi A, Qawas A. The prevalence and risk factors of obesity in the United Arab Emirates. Saudi J Obes. 2017:5(2):57 Available from: http://www.saudijobesity.com/text.asp?2017/5/2/57/221994. 
30. Haugen M, Brantsaeter AL, Winkvist A, Lissner L, Alexander J, Oftedal B, et al. Associations of pre-pregnancy body mass index and gestational weight gain with pregnancy outcome and postpartum weight retention: a prospective observational cohort study. 2014. Available from: http://www. biomedcentral.com/1471-2393/14/201

31. Ashley-Martin J, Woolcott C. Gestational Weight Gain and Postpartum Weight Retention in a Cohort of Nova Scotian Women. Matern Child Health J. 2014; 18(8):1927-35 Available from: http://www.ncbi.nlm.nih.gov/pubmed/24500210.

32. Lan-Pidhainy $X$, Nohr EA, Rasmussen KM. Comparison of gestational weight gain-related pregnancy outcomes in American primiparous and multiparous women. Am J Clin Nutr. 2013;97(5):1100-6 Available from: https://academic.oup.com/ajcn/article/97/5/1100/4577095.

33. Harris HE, Ellison GTH, Holliday M. Is there an independent association between parity and maternal weight gain? Ann Hum Biol. 1997;24(6):50719. Available from:. https://doi.org/10.1080/03014469700005272.

34. Weisman CS, Hillemeier MM, Symons Downs D, Chuang CH, Dyer AM. Preconception predictors of weight Gain during pregnancy. Prospective findings from the Central Pennsylvania Women's health study. Women's Heal Issues. 2010; 20(2):126-32. Available from:. https:/doi.org/10.1016/j.whi.2009.12.002.

35. Deputy NP, Sharma AJ, Kim SY, Hinkle SN. Prevalence and Characteristics Associated With Gestational Weight Gain Adequacy. Obstet Gynecol. 2015; 125(4):773-81.

36. Begum F, Colman I, McCargar LJ, Bell RC. On behalf of the Alberta pregnancy outcomes. Gestational weight Gain and early postpartum weight retention in a prospective cohort of Alberta women. J Obstet Gynaecol Can. 2012;34(7):637-47 Available from: https://www.sciencedirect.com/science/ article/pii/S1701216316353166?via\%3Dihub.

37. Olson CM, Strawderman MS. Modifiable behavioral factors in a biopsychosocial model predict inadequate and excessive gestational weight gain. J Am Diet Assoc. 2003;103(1):48-54 Available from: http://www.ncbi. nlm.nih.gov/pubmed/12525793.

38. Li N, Liu E, Guo J, Pan L, Li B, Wang P, et al. Maternal Prepregnancy body mass index and gestational weight Gain on offspring overweight in early infancy. PLoS One. 2013;8(10):1-9.

39. Agarwal MM, Dhatt GS, Punnose J, Koster G. Gestational diabetes: dilemma caused by multiple international diagnostic criteria. Diabet Med. 2005;22(12): 1731-6. Available from:. https://doi.org/10.1111/j.1464-5491.2005.01706.x.

40. Nguyen $\mathrm{CL}$, Pham NM, Binns CW, Van Duong D, Lee AH. Prevalence of gestational diabetes mellitus in eastern and southeastern Asia: A systematic review and meta-analysis. J Diabetes Res. 2018;2018:6536974.

41. Wahabi H, Fayed A, Esmaeil S, Alzeidan R, Elawad M, Tabassum R, et al. Riyadh mother and baby multicenter cohort study: the cohort profile. PLoS One. 2016;11(3):e0150297 Available from: http://www.pubmedcentral.nih. gov/articlerender.fcgi?artid $=4777404 \&$ tool=pmcentrez\&rendertype=abstract.

42. Okunoye G, Konje J, Lindow S, Perva S. Gestational diabetes in the Gulf region: streamlining care to optimise outcome. J Local Glob Heal Sci. 2015; 2(1):1-6. Available from:. https://doi.org/10.5339/jlghs.2015.2.

43. Zhu Y, Zhang C. Prevalence of gestational diabetes and risk of progression to type 2 diabetes: a global perspective. Curr Diab Rep. 2016;16(1):7. Available from:. https://doi.org/10.1007/s11892-015-0699-X.

44. Gao X, Yan Y, Xiang S, Zeng G, Liu S, Sha T, et al. The mutual effect of prepregnancy body mass index, waist circumference and gestational weight gain on obesity-related adverse pregnancy outcomes: a birth cohort study. PLoS One. 2017;12(6):1-13.

45. Boudet-Berquier J, Salanave B, Desenclos JC, Castetbon K Sociodemographic factors and pregnancy outcomes associated with prepregnancy obesity: effect modification of parity in the nationwide Epifane birth-cohort. BMC Pregnancy Childbirth. 2017;17(1):1-13.

46. Alberico S, Montico M, Barresi V, Monasta L, Businelli C, Soini V, et al. The role of gestational diabetes, pre-pregnancy body mass index and gestational weight gain on the risk of newborn macrosomia: Results from a prospective multicentre study. BMC Pregnancy Childbirth. 2014;14(1):23.

47. Shaw JE, Sicree RA, Zimmet PZ. Global estimates of the prevalence of diabetes for 2010 and 2030. Diabetes Res Clin Pract. 2010;87(1):4-14 Available from: http://www.ncbi.nlm.nih.gov/pubmed/19896746.

48. Torloni MR, Betrán AP, Horta BL, Nakamura MU, Atallah AN, Moron AF, et al. Prepregnancy BMI and the risk of gestational diabetes: a systematic review of the literature with meta-analysis: diagnostic in obesity and complications. Obes Rev. 2009;10(2):194-203.

49. Hung T, Hsieh T. Taiwanese Journal of Obstetrics \& Gynecology Pregestational body mass index, gestational weight gain, and risks for adverse pregnancy outcomes among Taiwanese women : A retrospective cohort study. Taiwan J Obstet Gynecol. 2016;55(4):575-81. Available from: https://doi.org/10.1016/j.tjog.2016.06.016.

50. Barisic T, Mandic V, Barac I. Associations of body mass index and gestational weight Gain with term pregnancy outcomes. Mater Socio Medica. 2017;29(1): 52 Available from: http://www.ejmanager.com/fulltextpdf.php?mno=262296.

51. Yang S, Peng A, Wei S, Wu J, Zhao J, Zhang Y, et al. Pre-pregnancy body mass index, gestational weight gain, and birth weight: a cohort study in China. PLoS One. 2015;10(6):1-12.

52. Dong B, Yu H, Wei Q, Zhi M, Wu C, Zhu X, et al. The effect of pre-pregnancy body mass index and excessive gestational weight gain on the risk of gestational diabetes in advanced maternal age. Oncotarget. 2017;8(35): 58364-71 Available from: http://www.oncotarget.com/fulltext/17651.

53. Vaswani PBL. Is obesity a significant risk predictor for adverse maternal and neonatal outcomes in morbidly obese pregnant women of Middle East countries? J Hypo Hyperglycemia. 2013;1:2-6.

54. Chung JGY, Taylor RS, Thompson JMD, Anderson NH, Dekker GA, Kenny LC, et al. Gestational weight gain and adverse pregnancy outcomes in a nulliparous cohort. Eur J Obstet Gynecol Reprod Biol. 2013;167(2):149-53. Available from:. https://doi.org/10.1016/j.ejogrb.2012.11.020.

55. Yee LM, Cheng YW, Inturrisi M, Caughey AB. Gestational weight loss and perinatal outcomes in overweight and obese women subsequent to diagnosis of gestational diabetes mellitus. Obesity. 2013;21(12):770-4.

56. Herring, S. J., \& Oken E. Obesity and diabetes in mothers and their children: can we stop the intergenerational cycle? Curr Diab Rep. 2010;11(1):1-13.

57. Robitaille J. Excessive gestational weight gain and gestational diabetes: importance of the first weeks of pregnancy. Diabetologia. 2015;58(10):2203-5.

58. Bogaerts A, Van den Bergh B, Nuyts E, Martens E, Witters I, Devlieger R. Socio-demographic and obstetrical correlates of pre-pregnancy body mass index and gestational weight gain. Clin Obes. 2012;2(5-6):150-9. Available from:. https://doi.org/10.1111/cob.12004.

59. Lee JM, Kim MJ, Kim MY, Han JY, Ahn HK, Choi JS, et al. Gestational weight gain is an important risk factor for excessive fetal growth. Obstet Gynecol Sci. 2014;57(6):442 Available from: https://synapse.koreamed.org/DOlx. php?id=10.5468/ogs.2014.57.6.442.

60. Athukorala C, Rumbold AR, Willson KJ, Crowther CA. The risk of adverse pregnancy outcomes in women who are overweight or obese. BMC Pregnancy Childbirth. 2010;10:56 Available from: http://www.ncbi.nlm.nih. gov/pubmed/20849609.

61. Hill B, Bergmeier H, McPhie S, Fuller-Tyszkiewicz M, Teede H, Forster D, et al. Is parity a risk factor for excessive weight gain during pregnancy and postpartum weight retention? A systematic review and meta-analysis. Obes Rev. 2017;18(7):755-64.

62. Hong SA, Peltzer K, Lwin KT, Aung LS. The prevalence of underweight, overweight and obesity and their related sociodemographic and lifestyle factors among adult women in Myanmar, 2015-16. PLoS One. 2018;13(3). https://doi.org/10.1371/journal.pone.0194454.

63. Hillemeier MM, Weisman CS, Chuang C, Downs DS, McCall-Hosenfeld J, Camacho F. Transition to overweight or obesity among women of reproductive age. J Women's Heal. 2011;20(5):703-10. Available from:. https://doi.org/10.1089/jwh.2010.2397.

64. Han YS, Ha EH, Park HS, Kim YJ, Lee SS. Relationships between pregnancy outcomes, biochemical markers and pre-pregnancy body mass index. Int J Obes. 2011;35(4):570-7. https://doi.org/10.1038/ijo.2010.162.

65. Rosato V, Edefonti V, Parpinel M, Milani GP, Mazzocchi A, Decarli A, Agostoni C, Ferraroni M. Energy contribution and nutrient composition of breakfast and their relations to overweight in free-living individuals: a systematic review. Adv Nutr. 2016;7(3):455-65. https://doi.org/10.3945/an.115.009548.

\section{Publisher's Note}

Springer Nature remains neutral with regard to jurisdictional claims in published maps and institutional affiliations. 